Revista Brasileira de

Engenharia Agrícola e Ambiental

v. 15, n.5, p.458-462, 2011

Campina Grande, PB, UAEA/UFCG - http://www.agriambi.com.br

agriambi

Protocolo 102.10 - 30/05/2010 • Aprovado em 03/03/2011

\title{
Eficiência de uso de nitrogênio em cobertura pelo feijoeiro irrigado
}

\author{
Edvaldo V. P. Sant'Ana ${ }^{1}$, Alberto B. dos Santos ${ }^{2} \&$ Pedro M. da Silveira ${ }^{2}$
}

\section{RESUMO}

Entre as técnicas de manejo necessárias para atingir alto potencial produtivo nos sistemas agrícolas irrigados, está a adubação nitrogenada. O objetivo deste trabalho foi avaliar os efeitos de doses de nitrogênio aplicadas em cobertura sobre a fitomassa e a eficiência de uso do nutriente pela cultivar BRS Horizonte de feijoeiro. O experimento foi conduzido na Embrapa Arroz e Feijão, em um Latossolo Vermelho distrófico, em delineamento experimental de blocos ao acaso, com quatro repetições e cinco doses de nitrogênio, 0, 30,60, 120 e $240 \mathrm{~kg} \mathrm{ha}^{-1}$, na forma de uréia, aplicadas em cobertura metade aos dez dias após a emergência (DAE) e a outra metade aos 17 DAE. A fitomassa no florescimento do feijoeiro relacionou-se linearmente com as doses de $\mathrm{N}$ aplicadas em cobertura, ao passo que a fitomassa na colheita apresentou resposta quadrática. As relações entre o índice de colheita de grãos e o índice de colheita de $\mathrm{N}$ com as doses de $\mathrm{N}$ foram quadráticas e os índices máximos foram estimados com a dose de $140 \mathrm{~kg} \mathrm{ha}^{-1}$ de N. A eficiência de uso de nitrogênio pelo feijoeiro variou com as doses de $\mathrm{N}$ aplicadas e com o tipo de eficiência calculada, enquanto as eficiências agronômica e fisiológica diminuíram com 0 incremento da dose.

Palavras-chave: Phaseolus vulgaris, matéria seca, eficiência agronômica, eficiência fisiológica

\section{The efficiency of use of nitrogen applied in top dressing in irrigated bean}

\begin{abstract}
$\mathrm{N}$ itrogen fertilization is one of the management techniques necessary to achieve high yield potential in irrigated agricultural systems. The objective of this study was to evaluate effects of nitrogen applied as topdressing on phytobiomass and nutrient use efficiency in dry bean cultivar BRS Horizonte. The experiment was conducted at the National Rice and Beans Research Center of Embrapa in a Distrophic Red Latosol in randomized block design with four replications. The nitrogen rates used were $0,30,60$, 120 and $240 \mathrm{~kg} \mathrm{ha}^{-1}$, half applied 10 days after plant emergence and half, 17 days after plant emergence in the form of urea. Dry bean phytobiomass was linearly related with $\mathrm{N}$ rate at flowering, while at harvest the response was quadratic. Relation between grain harvest index, nitrogen harvest index and $\mathrm{N}$ rate was quadratic and the maximum index was obtained at $140 \mathrm{~kg} \mathrm{~N} \mathrm{ha}^{-1}$. Nitrogen use efficiency by dry bean varied with $\mathrm{N}$ rate applied and type of efficiency while agronomical and physiological efficiencies decreased with increase $\mathrm{N}$ rates.
\end{abstract}

Key words: Phaseolus vulgaris, dry matter, agronomic efficiency, physiological efficiency

\footnotetext{
1 Instituto Federal de Educação, Ciência e Tecnologia do Tocantins, CEP 77021-090, Palmas, TO. Fone: (63) 3233 1329. E-mail: edvaldovps@bol.com.br

2 Embrapa Arroz e Feijão, CP 179, CEP 75375-000, Santo Antônio de Goiás, GO. Fone: (62) 3533 2153. E-mail: baeta@cnpaf.embrapa.br; pmarques@cnpaf.embrapa.br
} 


\section{INTRODUÇÃO}

Antes de seu caráter econômico, o feijão representa um alimento de alto significado social, devido ao hábito alimentar da população brasileira. O feijoeiro é uma planta bastante exigente em nutrientes e, em razão do ciclo curto, necessita de que eles estejam prontamente disponíveis nos momentos de maior demanda para não limitar a produtividade de grãos (Silva \& Silveira, 2000). Entre as técnicas de manejo necessárias para atingir alto potencial produtivo nos sistemas agrícolas irrigados, está a adubação nitrogenada. O nitrogênio $(\mathrm{N})$ promove a rápida expansão da folha e aumenta os componentes da produtividade. Em relação aos demais nutrientes, o $\mathrm{N}$ é absorvido em quantidades mais elevadas pelo feijoeiro. É responsável pelo incremento da área foliar da planta, o que aumenta a eficiência de intercepção da radiação solar, a taxa fotossintética e, consequentemente, a produtividade de grãos (Fageria \& Baligar, 2005).

Se não for adotado um manejo apropriado, poderá ocorrer deficiência de $\mathrm{N}$, que resulta em plantas com baixa fitomassa e senescência prematura, evidenciada pelo amarelecimento das folhas mais velhas. A eficiência de uso das fontes de $\mathrm{N}$ pelas culturas anuais, como o feijoeiro, é baixa, ao redor de 50\%, e as causas para esse baixo valor estão relacionadas com a dose e a época de aplicação inadequadas e associadas com a lixiviação, desnitrificação, imobilização e erosão do solo (Fageria \& Baligar, 2005) e difere com as cultivares (Furtini et al., 2006; Santos \& Fageria, 2007; Lago et al., 2009). Com isto, a estratégia de adubação de $\mathrm{N}$ deve ter, como objetivo, melhorar a sincronia entre a época de aplicação e a época de maior demanda pela planta, de forma a maximizar a absorção e a produtividade de grãos (Santos \& Fageria, 2008). A melhoria da eficiência de uso de $\mathrm{N}$ é desejável para aumentar a produtividade, reduzir os custos de produção e manter a qualidade ambiental.

Objetivou-se com este trabalho avaliar os efeitos de doses de nitrogênio aplicadas em cobertura sobre a fitomassa e a eficiência de uso do nutriente pela cultivar BRS Horizonte de feijoeiro.

\section{MATERIAL E MÉTODOS}

O experimento foi conduzido na área experimental da Embrapa Arroz e Feijão, na Fazenda Capivara, em Santo Antônio de Goiás, GO (16²8'00" S, 49 $17^{\circ}$ '00"'W e altitude de $823 \mathrm{~m}$ ), em solo classificado como Latossolo Vermelho distrófico, textura média, relevo suave, ondulado e fase floresta subcaducifólia/ cerrado. As análises químicas das amostras do solo coletadas no início do estudo, nas camadas de 0 a $0,10 \mathrm{~m}$ e 0,10 a $0,20 \mathrm{~m}$ de profundidade revelaram, respectivamente, 6,4 e 6,2 de $\mathrm{pH}$ em água (1:2,5); 34,3 e 26,1 $\mathrm{mmol} \mathrm{dm}^{-3} \mathrm{de} \mathrm{Ca} ; 14,1$ e 10,7 mmol $\mathrm{dm}^{-3} \mathrm{de} \mathrm{Mg} ; 16,5$ e 20,6 $\mathrm{mg} \mathrm{dm}^{-3} \mathrm{de}$ P; 92 e $78 \mathrm{mg} \mathrm{dm}^{-3}$ de K; 0,0

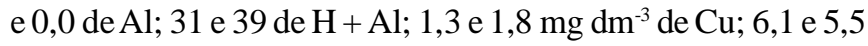
$\mathrm{mg} \mathrm{dm}{ }^{-3} \mathrm{de} \mathrm{Zn}$; 34 e $45 \mathrm{mg} \mathrm{dm}^{-3} \mathrm{de} \mathrm{Fe}$; 12 e $15 \mathrm{mg} \mathrm{dm}^{-3} \mathrm{de} \mathrm{Mn;} 21$ e $22 \mathrm{~g} \mathrm{~kg}^{-1}$ de matéria orgânica. Os resultados da composição granulométrica das amostras de solo na camada de 0 a $0,20 \mathrm{~m}$ de profundidade, foram $230 \mathrm{~g} \mathrm{~kg}^{-1}$ de argila; $280 \mathrm{~g} \mathrm{~kg}^{-1}$ de silte e $480 \mathrm{~g} \mathrm{~kg}^{-1}$ de areia. O fósforo e o potássio foram extraídos pelo extrator Mehlich $1\left(0,05 \mathrm{~mol} \mathrm{~L}^{-1} \mathrm{HCl}+0,0125 \mathrm{~mol} \mathrm{~L}^{-1} \mathrm{H}_{2} \mathrm{SO}_{4}\right)$ e determinados por colorímetro e fotômetro de chama, respectivamente. O cálcio $(\mathrm{Ca})$, o magnésio $(\mathrm{Mg})$ e o alumínio (Al) foram extraídos com solução de $1 \mathrm{~mol} \mathrm{~L}^{-1} \mathrm{KCl}$, sendo o Ca e o Mg determinados por titulação de EDTA e o Al por titulação de $\mathrm{NaOH}$. Os micronutrientes foram determinados com a mesma solução do $\mathrm{P}$, por espectrofotometria de absorção atômica, a matéria orgânica por oxidação via úmida e a análise granulométrica foi feita pelo método da pipeta. Os métodos de análise do solo usados neste estudo são descritos no manual de análise de solo, publicado pela EMBRAPA (Claessen, 1997).

A semeadura foi realizada no sistema plantio direto e o cultivo anterior foi milho em consórcio com braquiária, Brachiaria decumbens. No ano anterior, o feijoeiro foi cultivado também no sistema plantio direto, sendo a soja a cultura antecessora. Utilizou-se a cultivar BRS Horizonte de feijoeiro do grupo carioca, que apresenta as seguintes características: porte ereto, com 0,40 a $0,60 \mathrm{~m}$ de altura, hábito de crescimento indeterminado tipo II, flor branca, sementes opacas de cor bege com rajas marrons claras, com quatro a sete sementes por vagem e ciclo de 90 dias.

Previamente à instalação do experimento, a área foi dessecada com glifosate na dose de $2,16 \mathrm{~kg}$ i.a. (ingrediente ativo) ha-1 ${ }^{-1}$ Na semeadura do feijão foram colocadas 16 sementes por metro, em fileiras espaçadas $0,45 \mathrm{~m}$, o que resultou numa população de 300.000 plantas ha $^{-1}$, e a adubação empregada foi de $400 \mathrm{~kg} \mathrm{ha}^{-1}$ da fórmula 4-30-16, com base na análise do solo. A área experimental foi irrigada por aspersão, sistema pivô central, sendo as irrigações efetuadas sempre que a tensão de água do solo, na profundidade de 0 a $15 \mathrm{~cm}$, alcançava a faixa de $25 \mathrm{a} 40 \mathrm{kPa}$.

O delineamento experimental usado foi o de blocos ao acaso, com quatro repetições, e se avaliaram as doses de N: 0, 30, 60, 120 e $240 \mathrm{~kg} \mathrm{ha}^{-1}$, na forma de uréia, aplicadas em cobertura metade aos dez dias após a emergência (DAE) e o restante aos 17 DAE. O fertilizante foi distribuído manualmente em filete contínuo sobre a superfície do solo a cerca de $5 \mathrm{~cm}$ das plantas. As parcelas foram constituídas por oito linhas de feijoeiro de cinco metros de comprimento, espaçadas $0,45 \mathrm{~m}$, totalizando $18 \mathrm{~m}^{2}$. A área útil de cada parcela foi de $10,8 \mathrm{~m}^{2}$, sendo considerada uma bordadura de $0,5 \mathrm{~m}$ nas extremidades e nas laterais, resultando em seis linhas de quatro metros de comprimento. O controle de plantas daninhas foi realizado com a aplicação em pós-emergência do herbicida Fluazilop-p-butil + Fomesafen na dose de $250 \mathrm{~g}$ i.a. (ingrediente ativo) ha ${ }^{-1}$. Os demais tratos culturais foram realizados conforme as recomendações técnicas para a cultura.

$\mathrm{Na}$ floração, registrada aos 45 DAE, estádio R6, foram determinados o acúmulo de matéria seca de folhas (MSF) e o total da parte aérea (MST), quando da ocorrência da primeira flor aberta em 50\% das plantas. Por ocasião da colheita, foram determinados o acúmulo de MST e a produtividade de grãos, que foi usada no cálculo das eficiências de uso de N, as quais foram definidas empregando-se as seguintes fórmulas (Fageria et al., 2003):

$$
\mathrm{EA}=\left(\mathrm{PG}_{\mathrm{cf}}-\mathrm{PG}_{\mathrm{sf}}\right) /\left(\mathrm{QN}_{\mathrm{a}}\right)
$$


em que:

EA - eficiência agronômica, $\mathrm{kg} \mathrm{kg}^{-1}$

$\mathrm{PG}_{\mathrm{cf}}$ - produtividade de grãos com fertilizante nitrogenado $\mathrm{PG}_{\mathrm{sf}}$ - produtividade de grãos sem fertilizante nitrogenado $\mathrm{QN}_{\mathrm{a}}$ - quantidade de $\mathrm{N}$ aplicado, em $\mathrm{kg}$

$$
\mathrm{EF}=\left(\mathrm{PB}_{\mathrm{cf}}-\mathrm{PB}_{\mathrm{sf}}\right) /\left(\mathrm{AN}_{\mathrm{cf}}-\mathrm{AN}_{\mathrm{sf}}\right)
$$

em que:

$\mathrm{EF}$ - eficiência fisiológica, $\mathrm{kg} \mathrm{kg}^{-1}$

$\mathrm{PB}_{\mathrm{cf}}$ - produtividade biológica (palha e grãos) com fertilizante $\mathrm{N}$ $\mathrm{PB}_{\mathrm{sf}}$ - produtividade biológica sem fertilizante $\mathrm{N}$

$\mathrm{AN}_{\mathrm{cf}}$ - acumulação de $\mathrm{N}$ na parte aérea e grãos com fertilizante $\mathrm{N}$ $\mathrm{AN}_{\mathrm{sf}}$ - acumulação de $\mathrm{N}$ na parte aérea e grãos sem fertilizante $\mathrm{N}$

$$
\mathrm{EAF}=\left(\mathrm{PG}_{\mathrm{cf}}-\mathrm{PG}_{\mathrm{sf}}\right) / \mathrm{AN}_{\mathrm{cf}}-\mathrm{AN}_{\mathrm{sf}}
$$

em que:

EAF - eficiência agrofisiológica, $\mathrm{kg} \mathrm{kg}^{-1}$

$\mathrm{PG}_{\mathrm{cf}}$ - produtividade de grãos com fertilizante nitrogenado $\mathrm{PG}_{\mathrm{sf}}$ - produtividade de grãos sem fertilizante nitrogenado $\mathrm{AN}_{\mathrm{cf}}$ - acumulação de $\mathrm{N}$ na parte aérea e grãos com fertilizante $\mathrm{N}$ $\mathrm{AN}_{\mathrm{sf}}$ - acumulação de $\mathrm{N}$ na parte aérea e grãos sem fertilizante $\mathrm{N}$

O índice de colheita de grãos (ICG) e o índice de colheita de $\mathrm{N}$ (ICN), foram calculados pelas fórmulas:

$\mathrm{ICG}=$ Produtividade de grãos/produtividade de grãos + palha

$$
\begin{gathered}
\text { ICN = Acumulação de } \mathrm{N} \text { nos grãos/acumulação de N nos } \\
\text { grãos + palha }
\end{gathered}
$$

Determinou-se o teor de N na parte aérea e nos grãos pelo método microKjeldahl (Bremner \& Mulvaney, 1982). Os dados foram submetidos à análise da variância e, quando significativos, à análise de regressão.

\section{RESULTADOS E DISCUSSÃO}

Por ocasião da floração, foram quadráticas as respostas da massa da matéria seca de folhas (MSF) e total (MST) às doses de $\mathrm{N}$, sendo as máximas MSF e MST de 92 e $179 \mathrm{~g} \mathrm{~m}^{-2}$ obtidas com 184 e $192 \mathrm{~kg} \mathrm{ha}^{-1}$ de N, respectivamente (Figura 1A), indicando que a fitomassa no florescimento do feijoeiro, que ocorreu aos 45 DAE, se relaciona com as doses de $\mathrm{N}$ aplicadas em cobertura. Após a floração a planta inicia o processo de senescência, que se reflete, inicialmente, na paralisação da produção de matéria seca; a partir daí, começa a prevalecer a translocação, em substituição ao de crescimento.

A MST determinada na colheita ajustou-se ao modelo quadrático (Figura 1A); $140 \mathrm{~kg} \mathrm{ha}^{-1}$ de $\mathrm{N}$ foi a dose estimada pela equação de regressão para a obtenção de $617 \mathrm{~g} \mathrm{~m}^{-2}$ de fitomassa. No estudo de Santos \& Fageria (2007), a produtividade biológica das cultivares de feijoeiro teve desempenho diferente com o manejo de $\mathrm{N}$ em solo de várzea. De modo geral, esses autores verificaram maiores fitomassa e produtividade de grãos nas aplicações totais de $\mathrm{N}$ em sulcos
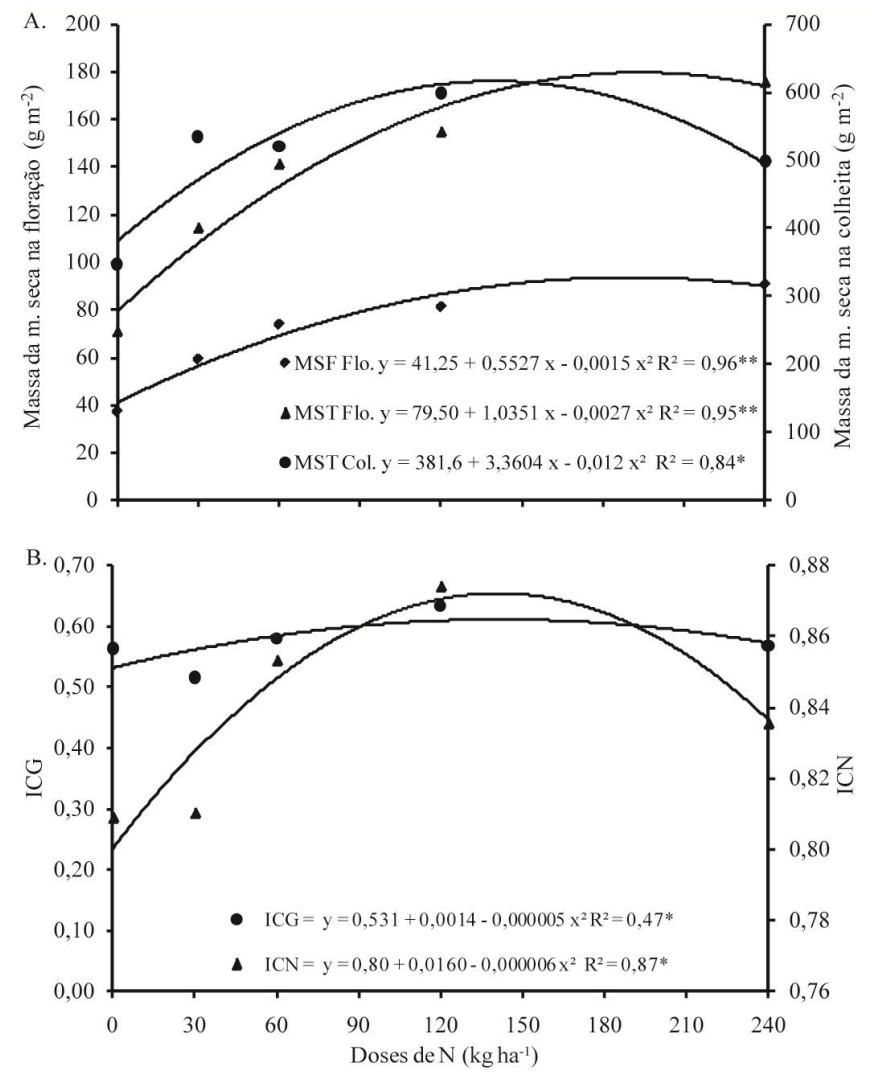

Figura 1. Relação entre dose de nitrogênio e massa da matéria seca de folha (MSF) e total (MST) na floração e total na colheita (A.) e índice de colheita de grãos (ICG) e índice de colheita de N (ICN) (B.) na cultivar BRS Horizonte de feijoeiro

distintos das linhas de semeadura, por ocasião desta ou aos 15 DAE. Soratto et al. (2001), verificaram efeito linear da aplicação de N em cobertura na MST do feijoeiro cultivado em sucessão ao milho em plantio direto.

O índice de colheita de grãos (ICG) e o índice de colheita de $\mathrm{N}$ (ICN) foram significativamente influenciados pelas doses de $\mathrm{N}$ e se ajustaram a modelos quadráticos; $140 \mathrm{~kg} \mathrm{ha}^{-1}$ de $\mathrm{N}$ foi a dose estimada pelas equações de regressão para a obtenção de 0,62 e 0,87 de ICG e ICN, respectivamente (Figura 1B). As variabilidades do ICG e do ICN devidas às doses de N, foram 47 e $87 \%$. Fageria \& Santos (2008) relataram correlação significativa e positiva de ICG e de ICN com a produtividade de grãos de feijão. Neste estudo, a produtividade máxima foi estimada com 0,65 e 0,88 de ICG e ICN, respectivamente, e as variações na produtividade de grãos relativas a esses índices foram de 48 e $68 \%$; portanto, valores muito próximos aos obtidos neste trabalho. Os autores mencionaram também que esses índices variam com os genótipos de feijoeiro e são influenciados pela fertilização nitrogenada. Ao avaliar a associação das frações nitrogenadas nas folhas com a eficiência no uso de $\mathrm{N}$ em linhagens de feijoeiro, Lago et al. (2009) verificaram que os teores de nitrogênio foram maiores sem a aplicação de $\mathrm{N}$, enquanto as demais frações de $\mathrm{N}$ e do $\mathrm{N}$-total nas folhas e nos grãos foram mais elevadas quando houve fornecimento de N. O índice de colheita de nutrientes expressa a proporção de nutrientes acumulada no grão e está associado 
à eficiência vegetal de utilização de nutrientes. A acumulação de alto nível de $\mathrm{N}$ é essencial para alta produtividade de grãos e, consequentemente, alto ICG (Sinclair, 1998). Com a dose de $140 \mathrm{~kg} \mathrm{ha}^{-1}$ de N, o feijoeiro produziu mais grãos por unidade de área foliar nos estudos de Santos \& Fageria (2008). Almeida et al. (1988), mencionam que um suprimento adequado de $\mathrm{N}$ estimula o aumento do tamanho potencial das vagens e das sementes. Incrementos do ICG e do ICN na cultura de arroz com aumento da dose de N foram obtidos por Fageria (2007). Segundo Sinclair (1998), há evidências de que o índice de colheita de várias culturas aumentou com a maior produtividade obtida nas últimas décadas e, assim, a seleção para ICG pode ser uma forma eficiente de aumentar a produtividade de grãos, especialmente em leguminosas. Araújo et al. (1999), verificaram pequena variabilidade para os índices de colheita de $\mathrm{N}$ e de $\mathrm{P}$ em genótipos de feijoeiro e constataram dificuldades para a seleção concomitante para altos teores de $\mathrm{N}$ e baixos teores de P nos grãos de feijão. De acordo com Fageria (1998), teoricamente a eficiência nutricional diminui com os níveis crescentes de um nutriente, caso tenha havido resposta da cultura, em virtude da diminuição relativa da produção com sucessiva adição de nutriente; assim, a melhor eficiência nutricional é aquela determinada sob nível de nutriente adequado em que a produtividade máxima é obtida.

Ocorreu efeito significativo da adubação nitrogenada em cobertura sobre a acumulação de N na folha (ANF), nos grãos (ANG) e total (ANT). A ANF aumentou linearmente com as doses de $\mathrm{N}$ com incremento de $0,008 \mathrm{~g} \mathrm{~m}^{-2}$ por quilograma de $\mathrm{N}$ (Figura 2A). Devido ao comportamento linear, não foi possível determinar sua resposta máxima ao fertilizante nitrogenado. A ANG e a ANT tiveram respostas quadráticas às doses de $\mathrm{N}$ e as máximas acumulações foram estimadas com as doses de, respectivamente, 157 e $162 \mathrm{~kg} \mathrm{ha}^{-1}$ de N. Os resultados estão de acordo com os reportados por Silveira \& Damasceno (1993), que observaram maior acumulação de $\mathrm{N}$ nas folhas do feijoeiro com o aumento das doses de $\mathrm{N}$ aplicadas ao solo.

A eficiência de uso de $\mathrm{N}$ pelo feijoeiro variou com as doses de $\mathrm{N}$ aplicadas e com o tipo de eficiência calculada. As eficiências agronômica (EA) e fisiológica $(\mathrm{EF})$ de $\mathrm{N}$ diminuíram com o incremento da dose de N (Figura 2B), enquanto a agrofisiológica (EAF) apresentou valores semelhantes, o que resultou, na média, em $24 \mathrm{~kg}$ de grãos produzidos por quilograma de $\mathrm{N}$ acumulado na planta, palha e grãos. Com 30 $\mathrm{kg} \mathrm{ha}^{-1}$ de $\mathrm{N}$ foram produzidos $27 \mathrm{~kg}$ de grãos por quilograma de $\mathrm{N}$ aplicado; na média, a EA foi de $16 \mathrm{~kg} \mathrm{~kg}^{-1}$, resultado esse similar ao da EA das cultivares de feijoeiro de ciclo médio em solo de várzea (Santos \& Fageria, 2007). Com esta mesma dose e com a acumulação de $1 \mathrm{~kg}$ de $\mathrm{N}$ na parte aérea e nos grãos, foram produzidos $63 \mathrm{~kg}$ de matéria seca; a média da $\mathrm{EF}$ foi de 30 $\mathrm{kg} \mathrm{kg}^{-1}$; a menor EF foi estimada com a dose de $200 \mathrm{~kg} \mathrm{ha}^{-1} \mathrm{de} \mathrm{N}$. A redução na eficiência de uso de $\mathrm{N}$ com o aumento das doses aplicadas também foi observada em outras culturas, como milheto (Heringer, 1995) e arroz irrigado (Fageria et al., 2009). Santos \& Fageria (2007) verificaram que a produtividade de grãos é positivamente associada às eficiências agronômica, de recuperação e de utilização de $\mathrm{N}$, as quais diferem com o manejo do fertilizante nitrogenado.
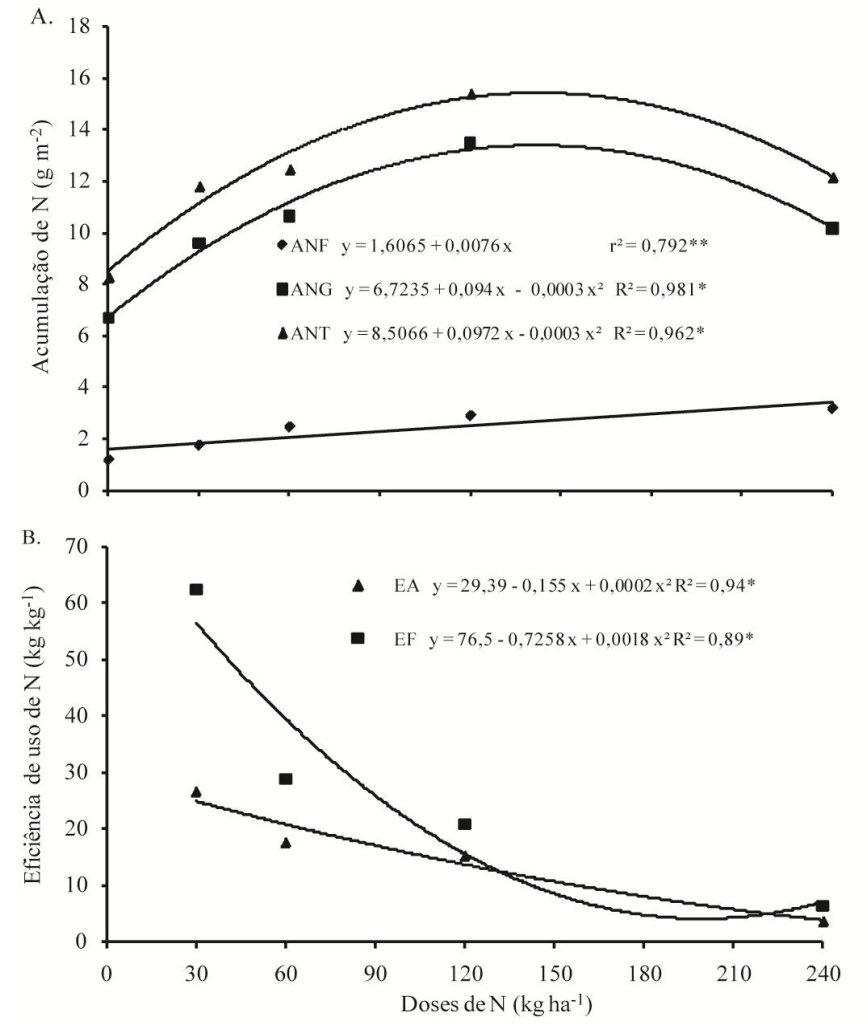

Figura 2. Relação entre dose de nitrogênio e acumulação de $N$ na folha (ANF), nos grãos (ANG) e total (ANT) (A.) e eficiência agronômica (EA) e eficiência fisiológica (EF) de N (B.) na cultivar BRS Horizonte de feijoeiro

Ao avaliar a eficiência na absorção e utilização de N pelas culturas da soja e do feijoeiro e por plantas daninhas, Procópio et al. (2004) observaram maior eficiência das raízes do feijoeiro na absorção de N. Ademais, constataram que um manejo inadequado desse nutriente nas culturas da soja e do feijoeiro pode agravar a competição com plantas daninhas.

\section{CONCLUSÕES}

1. Por ocasião do florescimento do feijoeiro a fitomassa relacionou-se linearmente com as doses de $\mathrm{N}$ aplicadas em cobertura, ao passo que na colheita a fitomassa apresentou resposta quadrática.

2. A eficiência de uso de nitrogênio pelo feijoeiro variou com as doses de $\mathrm{N}$ aplicadas e com o tipo de eficiência calculada e as eficiências agronômica e fisiológica diminuíram com o incremento da dose.

3. As relações entre o índice de colheita de grãos e o índice de colheita de $\mathrm{N}$ com as doses de $\mathrm{N}$ foram quadráticas e os índices máximos foram estimados com a dose de $140 \mathrm{~kg} \mathrm{ha}^{-1} \mathrm{de} \mathrm{N}$.

\section{LITERATURA CITADA}

Almeida, A. A. F.; Lopes, N. F.; Oliva, M. A.; Barros, R. S. Desenvolvimento e partição de assimilados em Phaseolus vulgaris submetido a três doses de nitrogênio e três níveis de luz. Pesquisa Agropecuária Brasileira, v.23, p.837-847, 1988. 
Araújo, A. P.; Lima, E. R. de; Oliveira, A. S. de; Dias, P. P.; Teixeira, M. G. Índices de colheita de nitrogênio e de fósforo de cultivares de feijoeiro. In: Reunião Nacional de Pesquisa de Feijão, 6, 1999, Salvador. Resumos... Santo Antônio de Goiás: Embrapa Arroz e Feijão, 1999. p.819-822.

Bremner, J. M.; Mulvaney, C. S. Nitrogen. In: Page, A. L. (ed.) Methods of soil analysis. 2. ed. Madison: American Society of Agronomy, 1982. p.595-624.

Claessen, M. E. C. (org.). Manual de métodos de análise de solo. 2.ed. Riode Janeiro: EMBRAPA-CNPS, 1997. 212p. Documentos, 1

Fageria, N. K. Otimização da eficiência nutricional na produção das culturas. Revista Brasileira de Engenharia Agrícola e Ambiental, v.2, p.6-16, 1998.

Fageria, N. K. Yield physiology of rice. Journal of Plant Nutrition, v.30, p.843-879, 2007.

Fageria, N. K.; Baligar, V. C. Enhancing nitrogen use efficiency in crop plants. Advances in Agronomy, v.88, p.97-185, 2005.

Fageria, N. K.; Santos, A. B. dos. Yield physiology of dry bean. Journal of Plant Nutrition, v.31, p.983-1004, 2008.

Fageria, N. K.; Santos, A. B. dos; Cutrim, V. dos A. Nitrogen uptake and its association with grain yield in lowland rice genotypes. Journal of Plant Nutrition, v.32, p.1965-1974, 2009.

Fageria, N. K.; Slaton, N. A.; Baligar, V. C. Nutrient management for improving lowland rice productivity and sustainability. Advances in Agronomy, v.80, p.63-152, 2003.

Furtini, I. V.; Ramalho, M. A. P.; Abreu, A. de F. B.; Furtini Neto, A. E. F. Resposta diferencial de linhagens de feijoeiro ao nitrogênio. Ciência Rural, v.36, p.1696-1700, 2006.

Heringer, I. Efeito de níveis de nitrogênio sobre a dinâmica de uma pastagem de milheto (Pennisetum americanum (L.) Leeke) sob pastejo. Santa Maria: UFSM, 1995. 133p. Dissertação Mestrado
Lago, F. J. do; Furtini Neto, A. E.; Furtini, I. V.; Ramalho, M. A. P.; Horta, I. de M. F. Frações nitrogenadas e eficiência nutricional em linhagens de feijoeiro (Phaseolus vulgaris L.). Ciência e Agrotecnologia, v.33, p.440-447, 2009.

Procópio, S. O.; Santos, J. B.; Pires, F. R.; Silva, A. A.; Mendonça, E. S. Absorção e utilização do nitrogênio pelas culturas da soja e do feijão e por plantas daninhas. Planta Daninha, v.22, p.365-374, 2004.

Santos, A. B. dos; Fageria, N. K. Características fisiológicas do feijoeiro em várzeas tropicais afetadas por doses e manejo de nitrogênio. Ciência e Agrotecnologia, v.32, p.2331, 2008.

Santos, A. B. dos; Fageria, N.K. Manejo do nitrogênio para eficiência de uso por cultivares de feijoeiro em várzea tropical. Pesquisa Agropecuária Brasileira, v.42, p.12371248, 2007.

Silva, C. C. da; Silveira, P. M. da. Influência de sistemas agrícolas na resposta do feijoeiro (Phaseolus vulgaris L.) irrigado à adubação nitrogenada em cobertura. Pesquisa Agropecuária Tropical, v.30, p.86-96, 2000.

Silveira, P. M. da; Damasceno, M. A. Doses e parcelamento de $\mathrm{K}$ e de $\mathrm{N}$ na cultura do feijoeiro irrigado. Pesquisa Agropecuária Brasileira, v.28, p.1269-1276, 1993.

Sinclair, T. R. Historical changes in harvest index and crop nitrogen accumulation. Crop Science, v.38, p.638-643, 1998.

Soratto, R. P.; Silva, T. R. B.; Arf, O.; Carvalho, M. A. C. Níveis e épocas de aplicação de nitrogênio em cobertura no feijoeiro irrigado em plantio direto. Cultura Agronômica, v.10, p.8999, 2001. 